\title{
HORNEANDO TRADICIÓN EN EL CONTEXTO DEL CAPITALISMO GLOBAL. LA ROSITA COMO REFERENTE DE LA IDENTIDAD ÍNTIMA EN LEÓN, GUANAJUATO
}

\author{
Baking tradition in the context of Global Capitalism. \\ La Rosita, as a benchmark for intimate identity in León, Guanajuato
}

\author{
Juana Edith Becerra Manrique \\ Universidad de Guanajuato, México \\ jedith_bm@hotmail.com
}

Recibido: $27-03-2018$

Aprobado: 28-05-2018

\begin{abstract}
Juana Edith Becerra Manrique es Licenciada en Historia del Arte por la Universidad de León. Maestra en Historia (Estudios Históticos Interdisciplinarioa) y candidata a Doctora en Ciencias Sociales en la Universidad de Guanajuato. Ganadora del Merito Universitario 2013 y 2014; becaria CONACyT, CONCyTEG y de la Dirección de Apoyo a la Investigación y el Posgrado (DAINPO) de la Universidad de Guanajuato.
\end{abstract}

\section{RESUMEN}

Enmarcada en una dinámica económica tendiente al capitalismo global permanece una panadería tradicional que conserva ciertas particularidades como son el proceso de elaboración y cocción del pan, donde se privilegia el trabajo manual del artesano panadero y el uso de leña en un horno abovedado de ladrillo comúnmente denominado "calabacero". La Rosita sigue alimentando a las familias leonesas que identifican a este pan como el tradicional de León y acuden cada noche a comprarlo y compartirlo en la cena con los suyos, haciendo del acto de comer un acto de socialización que posiblemente conserve rasgos de comensalía anteriores a las formas impuestas por el modelo hegemónico imperante en la localidad. A partir del diálogo histórico-antropológico y de la metodología ETNOAI ${ }^{1}$, nos acercamos a esta realidad social para dar cuenta de ella como una forma de resignificar el consumo del pan tradicional más allá de las definiciones desde una política económica hegemónica que no alcanza a abarcar todas sus categorías de análisis, pues toda práctica cultural debe contrastarse entre sí y frente a lo impuesto para no limitar su comprensión y valoración, la respuesta a su permanencia quizás esté en la identidad íntima de los consumidores del pan.

Palabras clave: capitalismo global; panadería tradicional; identidad íntima

\footnotetext{
${ }^{1}$ Etnología Audiovisual Interpretativa, propuesta metodológica de la Dra. Maricruz Romero Ugalde (Profesora Investigadora de la Universidad de Guanajuato).
} 


\begin{abstract}
Framed in an economic dynamic tending to the global capitalism, it remains a traditional bakery that preserves certain particularities such as the process of elaboration and baking of the bread, where the manual work of the artisan baker and the use of firewood in a domed brick furnace called "calabacero"- is favored. La Rosita continues feeding the families of León, Guanajuato, who identify this bread as the traditional one of this city. People go there every night to buy and share it at dinner with their family, making the act of eating an act of socialization that possibly preserves traits prior to the forms imposed by the prevailing hegemonic model in the locality. From the historical-anthropological dialogue and the ETNOAI methodology, we approach this social reality to account for it as a way of resignification of the consumption of traditional bread beyond definitions from a hegemonic economic policy that does not cover all of its categories of analysis, because every cultural practice must be contrasted with itself and with others and against what is imposed so their comprehension and assessment is not limited. Perhaps the answer to their permanence may be in the intimate identity of the bread consumers.
\end{abstract}

Keywords: global capitalism; traditional bakery; intimate identity

\title{
Rastreando a La Rosita
}

El consumo de pan en León, Guanajuato es una parte del legado español heredado del encuentro cultural que se remonta a mediados del siglo XVI cuando el virrey Antonio de Mendoza concedió mercedes para el poblamiento del territorio ubicado en el extremo sudoccidental del hoy Estado de Guanajuato. Fortín de paso entre los minerales de Zacatecas y Guanajuato, la Estancia de Señora fue creciendo hasta que el virrey Martín Enríquez de Almanza concretó la fundación de la villa en enero de 1576 (González Leal, 1990). Los barrios de indios se establecieron en San Miguel de la Real Corona y San Francisco del Coecillo; a su vez la población negra se asentó en el Barrio de Arriba, hoy Colonia Obregón. En la margen izquierda del Río de los Gómez, al noroeste del Centro Histórico y separado de éste por el boulevard Adolfo López Mateos surgió el Barrio de Santiago, consecuencia del tránsito entre el Coecillo y el Barrio Arriba ${ }^{2}$. En la zona central ${ }^{3}$ de Santiago (Padilla, 2002) está la

\footnotetext{
2 Es importante destacar el caso de estos dos barrios por ser en ellos donde gira la dinámica económica más significativa de la localidad y que tiene que ver con la producción del calzado. En el Barrio de Arriba se encuentran las tenerías donde se curten las pieles para la elaboración de diferentes productos como el zapato, cintos, chamarras, carteras, bolsas para dama, etcétera. Cruzando el Río de los Gómez y atravesando el Barrio de Santiago, está el Barrio del Coecillo, en él está la zona más grande de León para la venta de productos de piel, incluyendo el zapato. En el Barrio de Santiago podemos encontrar diferentes tiendas donde se vende piel, por lo tanto, este barrio es un lugar de paso obligado entre ambos Barrios.

3 Padilla hace una clasificación por zonas del Barrio de Santiago, una de ellas es la zona central donde se encuentran los sitios de referencia como el Mercado República, la parroquia de Santiago y la hoy desaparecida Arena Isabel; otra es la zona del Eje porque colinda con el Boulevard Adolfo López Mateos- principal avenida de la ciudad-, finalmente la zona de La Llamarada que recibe mayor influencia del Barrio Arriba y que se encuentra aledaña al Malecón del Río.
} 
panadería La Rosita, justo detrás del Mercado República, en la calle Artes y al costado derecho de lo que hasta el siglo pasado fue la Arena Isabel.

\section{La panadería tradicional}

Muchas panaderías tradicionales de León conservan características de aquéllas del siglo XVIII (García Acosta, 1989), como son el empleo de pocos trabajadores o artesanos panaderos -que privilegian el trabajo manual-, bajo las órdenes de un patrón dueño de panadería y de los instrumentos de trabajo. El uso de tecnología se limita a batidoras eléctricas y algunos instrumentos simples para el cortado y labrado del pan; mientras que la producción de pan satisface las necesidades de los vecindados en torno a la panadería. Para la cocción se utilizan hornos de gaveta, eléctrico y el abovedado ${ }^{4}$; todos funcionan a base de gas, excepto por el uso de leña que se emplea en el horno calabacero de La Rosita.

Desde 1998, el Instituto Nacional de Estadística y Geografía (INEGI) cuenta con el Sistema de Clasificación Industrial de América del Norte (SCIAN) para las unidades económicas en México derivado de la firma del Tratado de Libre Comercio (TLC) con Estados Unidos y Canadá, en él la panadería tradicional corresponde al tipo de:

\footnotetext{
Unidades económicas dedicadas principalmente a la elaboración y venta directa al público en general de una gran variedad de productos frescos de panadería, como pan, pasteles y pastelillos. Estas unidades económicas también pueden distribuir los productos que elaboran a otras unidades económicas, entregándolos en diferentes formas, como pan en charolas, cajas, canastos. Los productos individuales pueden estar envueltos en papel o bolsas de plástico, pero no están empacados de acuerdo con la normatividad preestablecida (INEGI, 2013)5.
}

Esta definición sin embargo, no hace mención de otras características que también podríamos considerar como unidades de análisis referentes a la panadería tradicional. Es por esto que el registro audiovisual nos permitió un acercamiento más profundo a esas otras características que no son mencionadas por el SCIAN, pero que son relevantes para dar cuenta de la forma en cómo se elabora el pan artesanal y tener el testimonio de los actores involucrados en esta realidad social. Partiendo de esto surgen las interrogantes ¿Cómo funciona una panadería tradicional? ¿Qué elementos la hacen diferente de otras también

\footnotetext{
4 También llamado de ladrillo, de mampostería o calabacero.

${ }^{5}$ www.inegi.org.mx, consultado el 28 de julio de 2013.
} 
tradicionales? ¿Por qué los leoneses siguen consumiendo pan elaborado tradicionalmente y porqué en La Rosita?

\section{La experiencia ETNOAI}

El acercamiento a ETNOAI nos permitió el conocimiento de una nueva perspectiva metodológica para aproximarnos a la realidad social objeto de estudio, esta propuesta jerarquiza al ser humano quien, mientras participa de la producción de un documental, se refrenda como ser profesional e identifica su compromiso social en el ejercicio mismo que conlleva la investigación (Romero, 2011). Este compromiso se concretó en el momento que estuvimos en la panadería y pudimos percatarnos de las características que la hacen ser tradicional, las expectativas previas a lo que entendíamos por "tradicional" fueron rebasadas una vez que identificamos otras variables que hasta el momento no habíamos encontrado en alguna otra fuente, incluyendo a las expresadas en el SCIAN.

Además, el registro audiovisual de las entrevistas semi-estructuradas a los consumidores y al artesano panadero, nos permitió dar voz a los sujetos de estudio, su discurso, sus gestos y sus énfasis fueron rescatados gracias al uso de las herramientas tecnológicas insertas en el lenguaje cinematográfico permitiendo una interacción más directa entre ellos y los futuros espectadores, abriendo así un canal de comunicación menos manipulado por el investigador (Romero, 2011). En este sentido podemos decir que, aunque la edición del video rescata los aspectos más importantes del discurso de los actores sociales ${ }^{6}$, la experiencia ETNOAl fue relevante para intentar mostrar al público el contexto en el que se desarrolla esta realidad social, es decir, la filmación del panadero en su lugar de trabajo y de los consumidores haciendo fila para comprar el pan, es un intento por acercar al público de forma más directa al ambiente donde éstos se desarrollan. El discurso audiovisual que construimos pretende dar cuenta de la escenografía como punto de referencia para el espectador, quien, a través del video pueda ver al artesano y los comensales del pan desarrollándose en su propio contexto, donde el registro de su lenguaje corporal y gestual también dan cuenta de su sentir y su pensar, de tal suerte que

\footnotetext{
${ }^{6}$ No debemos omitir que el ejercicio interpretativo del investigador está implícito a la hora de la selección del material y la construcción del discurso narrativo que se hizo a partir de esto, sin embargo, la presentación del video-documental ayudan al público a insertarse en el entorno donde interactúan los actores sociales, entonces, la descripción del contexto es más directa.
} 
el actor social no sólo es actor de esa realidad, sino que se convierte en protagonista de su realidad.

También es importante mencionar que ETNOAI no sólo enriqueció el trabajo etnográfico, sino que a partir del manejo técnico, el conocimiento de la historia del cine, la elaboración de un guión literario y de una carpeta de producción nos llevaron a conocer una nueva forma de hacer investigación social que se convirtió en una alternativa metodológica que nos comprometió a presentar resultados a través de la conclusión de un video. El laboratorio integra alumnos de licenciatura y posgrado, en este caso, la colaboración entre un estudiante de la Licenciatura en Antropología Social y del Doctorado en Ciencias Sociales ${ }^{7}$ permitieron el desarrollo de las capacidades eje de esta propuesta: autonomía, trabajo en equipo y el manejo de herramientas; las tres enfocadas al desarrollo del ser profesional, refrendando así el compromiso social que como investigadores debemos estar siempre reflexionando (Romero, 2011).

\section{Horneando tradición}

En La Rosita, al igual que las panaderías del siglo XVIII descritas por García Acosta también funciona como vivienda (1989), en ella habita el dueño de la panadería quien es el hermano menor de una familia de panaderos. Es una casa de $5 \mathrm{~m}$ de ancho y $30 \circ 35 \mathrm{~m}$ de largo, aproximadamente, la primera habitación sirve para el expendio del pan, tiene anaqueles de metal dispuestos alrededor de la misma para que los consumidores recojan el pan con pinzas y en unas charolas que también son de metal. Tiene también un mostrador donde generalmente está el dueño de la panadería quien cuenta y cobra las piezas de pan, además hay una televisión y un pequeño refrigerador donde se guarda la leche que ocasionalmente los consumidores compran ahí. Después, y a lo largo de la casa, están las habitaciones que sirven de vivienda al hermano menor, como son recamara, baño y cocina; al final del pasillo está el amasijo, en él hay una mesa grande de trabajo elaborada de madera y con dos niveles, el superior sirve para amasar y el inferior para guardar instrumentos y materias primas, también hay una artesa $^{8}$ donde se amasa la mezcla para el bolillo -que únicamente sale los domingos por las mañanas- y de las conchas -piezas de mayor demanda que requieren una mayor cantidad de masa para elaborarlas-.

\footnotetext{
${ }^{7}$ Ambos de la Universidad de Guanajuato, aunque ETNOAl también recibe a estudiantes de intercambio de otras universidades.

8 Es una especie de cajón de madera que mide aproximadamente $60 \mathrm{~cm}$ de ancho por $2 \mathrm{~m}$ de lago.
} 
Alrededor del amasijo y dispuestos en las paredes hay tableros de madera que sirven para el guardado de materias primas y algunas herramientas de trabajo, asimismo hay anaqueles de metal que sirven para poner las charolas que contienen la masa en reposo mientras se fermenta, o bien, el pan ya labrado listo para hornearse. También alrededor y en el piso podemos encontrar costales de harina, botes con mantequilla, piloncillo, azúcar, etcétera.

Detrás del amasijo está un patio cuyo techo está cubierto con lámina, pero dejando un espacio libre para la circulación del aire y la salida del humo que se genera a la hora de quemar la leña en el horno. En él hay dos hornos de mampostería, uno de 50 años y en desuso por estar "caído"; el otro en función y mide alrededor de $2.5 \mathrm{~m}$ de ancho por $2.5 \mathrm{~m}$ de profundidad, el orificio que hace las veces de "portal" para meter el pan está como $1.50 \mathrm{~m}$ de altura y mide 60 × 60 centímetros; se mantiene caliente en todo momento -incluso durante la noche cuándo no se cose el pan-. La temperatura del horno es regulada con mayor o menor cantidad de leña, ésta se coloca en la parte izquierda del horno con una lámina de metal que la cubre y evita el contacto directo con el pan previniendo que se queme, ocasionalmente se mete un bote de aluminio lleno de agua para minimizar la producción de humo; además el panadero "regula" la temperatura del horno por medio de su propia mano, la cual introduce de manera intermitente para "medir" el calor e ir metiendo en un determinado orden las diferentes piezas de pan según su proceso de cocción. El patio también funge como lugar de almacenamiento para la leña, en él hay algunos tableros de metal multifuncionales y que se usan en las diferentes etapas de proceso de elaboración del pan, una especie de mesa de madera donde se aplasta el piloncillo con una piedra para las chorreadas y las palas de madera que sirven para meter y sacar las charolas con el pan. Ambos, tanto el amasijo como el patio donde se encuentra el horno, tienen piso y paredes de cemento, éstas últimas dan señales de desgaste en la pintura, además de que están manchadas de negro por el hollín generado del proceso de horneado.

Esta panadería es atendida por una familia y la división del trabajo es simple y está en función de los roles que cada uno de sus miembros desempeña: el despachador del pan es el hermano menor que conoce el proceso productivo pero se encarga de atender a los consumidores, contar y cobrar el pan; el hermano mayor es el artesano panadero quien ejecuta de forma artesanal todo el proceso de elaboración del pan, es decir, se encarga del cernido, pesado, amasado, sobado, apuñado, labrado, cortado y horneado del mismo. Hay otro panadero cuñado de ambos hermanos, él se encarga de elaborar las galletas y 
polvorones, así como de acarrear el pan a la habitación que hace las veces de expendio, también limpia las charolas. Ambos panaderos descansan los domingos, ese día hace el pan un sobrino de ambos, pero hijo de otro hermano. Eventualmente están los hijos del artesano panadero quienes también ayudan a trasladar el pan del amasijo al expendio e incluso salen a la calle para ofertarlo en canastos. Los panaderos cumplen con una jornada laboral que se extiende de 12 a 14 horas, generalmente empiezan a trabajar desde las 8 o 9 de la mañana y terminan hasta las 10 u 11 de la noche, dependiendo de la temperatura ambiental y el tiempo que inviertan para la elaboración del pan, pues éste es siempre fluctuante porque la tecnología que utilizan es simple, por lo tanto el proceso es totalmente manual, por ser las manos del artesano sus principales herramientas.

Como ya mencionamos antes, esta panadería conserva algunas características de las panaderías del siglo XVIII descritas por García Acosta, como son la dupla casa/panadería, la distribución del espacio físico del amasijo y las características del horno, sin embargo, existen diferencias como son el tipo de herramientas y la organización del trabajo, pues en La Rosita prevalece completamente el carácter manual del proceso de elaboración del pan y una familia se encarga de ella, sin que exista una total diferencia entre los dueños de panadería y sus trabajadores. Por otro lado, carece de los elementos propios de una organización manufacturera como en las panaderías dieciochescas, es decir, la concentración de los medios de producción no está en manos de un capitalista; no hay una figura clara del patrono que se exime del trabajo manual y que confíe las funciones de dirección y vigilancia directa de los obreros a una categoría especial de empleados asalariados -en La Rosita no existe esta última figura, pues no hay quien vigile a los panaderos-; no se emplean de forma simultánea a obreros y no hay división de trabajo en función de esto; además que la jerarquización del trabajo está definida más bien por los roles que los hermanos desempeñan en la panadería (1989).

En cuanto al uso de tecnología, sabemos que hoy día ésta puede sustituir algunas tareas del proceso de producción como son el amasado y horneado del pan, sin embargo en La Rosita prevalece el trabajo netamente manual, conservando así el carácter artesanal y generando una dinámica muy particular en lo que respecta al horario de venta, el cual sólo es de 7 a 10 de la noche, incluso la "salida" del pan al expendio no es siempre la misma pues varía por las limitaciones implícitas en el trabajo manual; lo mismo sucede en cuanto al volumen de pan que se produce para ser vendido, éste es menor en 
comparación con otras panaderías de la localidad. Podríamos decir que esta panadería no está en consonancia con la dinámica económica imperante en la ciudad de León, la cual se inserta en el modelo económico tendiente a la globalización. Sin embargo, y a pesar de ello, los leoneses identifican a ésta como una panadería tradicional y acuden a ella muchas veces sin importar la lejanía entre ésta y sus viviendas, o bien tolerando el "retraso" del pan aunque se viva cerca de ella.

\section{La Rosita como referente identitario en el contexto del capitalismo global}

La Rosita cumple con las características marcadas por el SCIAN para la panadería tradicional, éstas se ajustan a los estándares internacionales una vez que México firmó el TLC, entrando con ello en una política económica transnacional y por tanto a un nuevo sistema global que impulsa procesos ideológicos, políticos, sociales, económicos y culturales del mundo actual y que intervienen en nuestras biografías individuales y de grupo (Robinson, 2007). Así, se generan nuevas formas de interacción social que se ajustan a las nuevas demandas y dependencias de alcance mundial, donde México tiene que "amoldarse" a patrones establecidos hegemónicamente y desde el norte, de acuerdo a la vertiente teórica del capitalismo global mencionada por Robinson y donde Estados Unidos funge como el único regidor (2007). El mismo autor plantea la tesis globalista del mundo capitalista donde ya no sólo se sostiene en el poderío un solo Estado imperante, o bien donde el capital global sea el que domine, sino que menciona: "Estamos en un momento histórico de transición que envuelve con frecuencia la interpretación conflictiva de viejas y nuevas formas" (Robinson, 2007, pp.13-14), donde lo tradicional convive con lo global, al menos así ocurre en el caso de esta panadería contextualizada en la ciudad de León.

Sin embargo, tales características están rebasadas y la pervivencia de lo tradicional desafía la tesis del capitalismo global hegemónico que se entromete en los cambios de las prácticas alimentarias de los individuos como agentes sociales, homogeneizando con ello patrones de conducta que responden a una compleja dinámica de ahorro del tiempo que se invierte en la comensalía colectiva para tratar de eliminarla en beneficio de la clase capitalista y, aunque dominante, cuestionable desde el punto de vista de los consumidores que prefieren acudir a La Rosita a comprar una pieza de pan no sólo por su olor, sabor, consistencia o por el simple hecho de cenar "algo" antes de dormir, sino 
por los sentimientos que les genera el comer una pieza de pan elaborada de manera artesanal.

Posiblemente sea en el consumo donde puede estar la respuesta a la permanencia de la panadería tradicional en esta ciudad, porque en ello se considera la identidad referida como una narrativa personal, biográfica (Giménez, 2009), en fin... íntima, que remonta al ser a su pasado inmediato, el cual actualiza a la hora de consumir el pan y por tanto, el comerlo "reviste de significado social una actividad básica; con tanto significado, en realidad, que casi puede llegar a perderse de vista la actividad misma" (Mintz, 1996, p. 27). Esta identidad íntima, enmarcada en una sociedad compleja con una evidente tendencia al capitalismo global, es también punto de referencia y anclaje nostálgico de la niñez cuando los consumidores describen sus sentires a la hora de comer el pan de La Rosita con su familia, pues en su práctica rememoran aquello que les fue grato en su infancia y donde podríamos encontrar la pauta que nos permita identificar lo emblemático en el consumo del pan en esta panadería y no en otra, porque "lo emblemático [...] no necesariamente tiene que ver con la historia del proceso ritual, sino con lo memorable para sentirse identificados con un lugar específico" (Romero, 2012, p.34).

Los consumidores de pan leoneses siempre hacen referencia a La Rosita como una panadería tradicional; algunos la conocen por recomendación y muchos otros acuden a ella desde niños cuando sus padres llevaban el pan para cenar a la hora del término de la jornada laboral y el comienzo de la socialización familiar en los comedores de aquellas familias, nucleares y extensas -al mismo tiempo tan alejadas y cercanas a la realidad actual-. Los hijos de esos padres, ahora cabezas de sus propias familias nucleares, eventualmente acuden a comprar pan, no sólo a esta panadería sino a muchas otras ${ }^{9}$, una vez que la salida del trabajo -cualquiera que éste sea- lo permita. Comprar pan en La Rosita les genera referentes significativos más allá del puro hecho de comer, donde éstos son simbólicos y se los comunica simbólicamente; también tienen su historia (Mintz, 1996, p.28), historia que siempre tiene un halo de nostalgia en la visión y el discurso de los comensales del pan que vivimos en la ciudad de León.

\footnotetext{
9 En la ciudad de León, son más las panaderías que podríamos caracterizar como insertas en la dinámica económica imperante, donde la industrialización tiene cabida principalmente por la incorporación de tecnología en el proceso de elaboración del pan.
} 


\section{Conclusiones}

En lo aquí descrito se pretende dar cuenta de que lo tradicional va más allá de los paradigmas establecidos hegemónicamente y su consumo está relacionado con hábitos de consumo que van más allá del capitalismo global, porque los leoneses continúan comprando el pan en La Rosita donde los procesos se particularizan en comparación con otras panaderías tradicionales y se muestra como una definición propuesta desde una política económica no siempre es suficiente para abarcar todas las categorías de análisis de cualquier realidad social que emerja de la cultura.

La discusión a propósito de referentes identitarios y emblemáticos que se generan a partir de la panadería tradicional podrían explicarse a través del discurso de los consumidores de pan, quienes identifican a La Rosita como un ejemplo de ello. El hecho de reconocer a esta panadería por encima de otras de la localidad, es lo que nos da cuenta de la construcción de identidad en torno a ésta y diferente de la identidad leonesa a partir de su principal actividad económica: la producción del calzado.

Ahora bien, lo tradicional va más allá de los paradigmas establecidos hegemónicamente y de la conservación de procesos de producción heredados del pasado -atreviéndonos un poco, quizás desde tiempos de la Colonia-. Las formas de consumo también prevalecen, porque los leoneses continúan comprando el pan en La Rosita, una panadería que es testimonio de procesos de elaboración del pan diferentes e incluso contrarios a la dinámica comercial imperante de hoy día. Cuando los mercados buscan expandirse más allá de las fronteras nacionales, la venta de pan en esta panadería sólo satisface las necesidades de pocos comensales de la localidad, quienes además siguen acudiendo a ella por tradición familiar y porque en ello va implícito el rememorar una práctica cotidiana e íntima que se actualiza cuando se lleva el pan a la mesa para cenar. Por lo tanto, es pertinente decir que puede considerarse al consumo de pan en La Rosita como un referente identitario de esta ciudad. 


\section{Agradecimientos}

A la panadería La Rosita, que me abrió sus puertas para indagar sobre el trabajo que ahí se realiza día con día; a la División de Ciencias Sociales y Humanidades del Campus León de la Universidad de Guanajuato por permitirme ser parte del programa académico del Doctorado en Ciencias Sociales; a la Dra. Maricruz Romero Ugalde por su acompañamiento y dirección durante esta investigación; a la Dra. Carlota Meneses por sus comentarios; a David Tafoya por su invaluable colaboración en la edición del vídeo para ETNOAl y a Sergio Barbosa Macías por su amistad y por ser mi corrector de estilo en inglés.

\section{BIBLIOGRAFÍA}

García Acosta, Virginia. (1989). Las panaderías, sus dueños y trabajadores. Ciudad de México, siglo XVIII. México: Ediciones de la Casa Chata.

Giménez, Gilberto. (2009). Identidades sociales. México: Consejo Nacional para la Cultura y las Artes e Instituto Mexiquense de Cultura.

González Leal, Mariano. (1990). León, Trayectoria y destino. León, Guanajuato: Ayuntamiento Municipal de León, Guanajuato 1989-1991.

Instituto Nacional de Estadística y Geografía. México. www.inegi.org.mx Consulta: 28 de julio de 2013.

Mintz, Sidney W. (1996). Sabor a comida, sabor a libertad, Invenciones en la comida, la cultura y el pasado. México: Consejo Nacional para la Cultura y las Artes, Centro de Investigaciones y Estudios Superiores en Antropología Social y Editorial La Reina Roja.

Padilla González del Castillo, Ernesto. (2002). Identidad y Paisaje Urbano, El caso del barrio de Santiago. Tesis para obtener grado de Maestría en Arquitectura. León, Guanajuato: Universidad Iberoamericana.

Robinson, William I. (2007). Una teoría sobre el capitalismo global, Producción, clases y Estado en un mundo transnacional. Colombia: Ediciones Desde Abajo. 
Romero Ugalde, Maricruz. (2011). "Etnografía Audiovisual Interpretativa (Etnoai): una propuesta teórico-metodológica en el marco de la antropología visual". En: Inventario antropológico 9 (2007-2008): pp. 222-229. México: Universidad Autónoma Metropolitana.

Romero Ugalde, Maricruz. (2012). Entre la Guerra y la Paz, El ritual y la construcción de las identidades. USA: Editorial Académica Española. 